\title{
What is the minimal cardinal of a family which shatters all $d$-subsets of a finite set?
}

\author{
N. Chevallier and A. Fruchard
}

March 5, 2022

In this note, $d \leq n$ are positive integers. Let $S$ be a finite set of cardinal $|S|=n$ and let $2^{S}$ denote its power set, i.e. the set of its subsets. A $d$-subset of $S$ is a subset of $S$ of cardinal $d$. Let $\mathcal{F} \subseteq 2^{S}$ and $A \subseteq S$. The trace of $\mathcal{F}$ on $A$ is the family $\mathcal{F}_{A}=\{E \cap A ; E \in \mathcal{F}\}$. One says that $\mathcal{F}$ shatters $A$ if $\mathcal{F}_{A}=2^{A}$. The $V C$-dimension of $\mathcal{F}$ is the maximal cardinal of a subset of $S$ that is shattered by $\mathcal{F}$ [7]. The following is well-known [7, 4, 5]:

Theorem 1. (Vapnik-Chervonenkis, Sauer, Shelah)

If $\operatorname{VC}$-dim $(\mathcal{F}) \leq d$ (i.e. if $\mathcal{F}$ shatters no $(d+1)$-subset of $S$ ) then $|\mathcal{F}| \leq c(d, n)$, where

$$
c(d, n)=\left(\begin{array}{l}
n \\
0
\end{array}\right)+\cdots+\left(\begin{array}{l}
n \\
d
\end{array}\right) .
$$

Moreover this bound is tight: It is achieved e.g. for $\mathcal{F}=\left(\begin{array}{c}S \\ \leq d\end{array}\right)$, the family of all $k$-subsets of $S$, $0 \leq k \leq d$

A first natural question is:

Question 1. Assume a family $\mathcal{F} \subseteq 2^{S}$ is maximal for the inclusion among all families of $V C$-dimension at most $d$. Does $\mathcal{F}$ always have the maximal possible cardinal $c(d, n)$ ?

Let us define the index of $\mathcal{F}$ as follows:

$$
\text { Ind } \mathcal{F}=\max \{d \in\{0, \ldots, n\} ; \mathcal{F} \text { shatters all } d \text {-subsets of } S\} \text {. }
$$

Let $C(d, n)=\min \{|\mathcal{F}| ;$ Ind $\mathcal{F}=d\}$. For instance, we have $C(1, n)=2$, with the (only possible) choice $\mathcal{F}=\{\emptyset, S\}$. Of course we have $2^{d} \leq C(d, n) \leq 2^{n}$. The question is:

Question 2. Give the exact value of $C(d, n)$ for $2 \leq d \leq n$. If this is not possible, give lower and upper bounds as accurate as possible.

A well-known duality yields another formulation of Question 2 , Let $\varphi: S \rightarrow 2^{\mathcal{F}}, a \mapsto\{E \in$ $\mathcal{F} ; a \in E\}$ and set $\mathcal{S}=\varphi(S)$. In this manner, we have for all $a \in S$ and all $E \in \mathcal{F}$ :

$$
a \in E \Leftrightarrow E \in \varphi(a)
$$

One can check that $\mathcal{F}$ shatters $A \subseteq S$ if and only if, for every partition $(B, C)$ of $A$ (i.e. $A=B \cup C$ and $B \cap C=\emptyset)$ the intersection $\left(\bigcap_{b \in B} \varphi(b)\right) \cap\left(\bigcap_{c \in C} \overline{\varphi(c)}\right)$ is nonempty, where the notation $\bar{Y}$ stands for $\mathcal{F} \backslash Y$.

If Ind $\mathcal{F} \geq 2$, then $\varphi$ is a one-to-one correspondance from $S$ to $\mathcal{S}$, hence we have $\log n \leq$ $C(d, n)$ for all $2 \leq d \leq n$, where $\log$ denotes the logarithm in base 2 . 
The case $d=2$. Using for instance the binary expansion, it is easy to show that the order of magnitude of $C(2, n)$ is actually $\log n$. The next statement refines this.

Proposition 2. If $n=\frac{1}{2}\left(\begin{array}{c}2 l \\ l\end{array}\right)=\left(\begin{array}{c}2 l-1 \\ l-1\end{array}\right)$, then $C(2, n)=2 l$.

Proof. (Recall the notation $\bar{A}=\mathcal{F} \backslash A$.) We first prove by contradiction that $C(2, n)>2 l-1$. Actually, if a family $\mathcal{F}$ of subsets of $S$ shatters all 2-subsets of $S$, then the image $\mathcal{S} \subseteq 2^{\mathcal{F}}$ of $S$ by $\varphi$ must satisfy

$$
\forall A \neq B \in \mathcal{S}, A \cap B, A \cap \bar{B}, \bar{A} \cap B \text {, and } \bar{A} \cap \bar{B} \text { are nonempty. }
$$

In particular $\mathcal{S}$ is a Sperner family of $\mathcal{F}$ (i.e. an antichain for the partial order of inclusion; one finds several other expressions in the literature: 'Sperner system', 'independent system', 'clutter', 'completely separating system', etc.). For a survey on Sperner families and several generalizations, we refer e.g. to [1] and the references therein.

Assume now that $|\mathcal{F}|=2 l-1$; it is known [6, 2, 3] that all Sperner families of $\mathcal{F}$ have a cardinal at most $\left(\begin{array}{c}2 l-1 \\ l-1\end{array}\right)$, and that there are only two Sperner families of maximal cardinal: the families $\left(\begin{array}{c}\mathcal{F} \\ l-1\end{array}\right)$ and $\left(\begin{array}{c}\mathcal{F} \\ l\end{array}\right)$, i.e. of $(l-1)$-subsets, resp. l-subsets of $\mathcal{F}$. However, none of these families satisfies both $A \cap B$ and $\bar{A} \cap \bar{B}$ nonempty in (2). As a consequence, we must have $|\mathcal{F}| \geq 2 l$.

Conversely, let $S=\left\{a_{1}, \ldots, a_{n}\right\}$, consider $\left(\begin{array}{c}\{1, \ldots, 2 l\} \\ l\end{array}\right)$, the set of $l$-subsets of $\{1, \ldots, 2 l\}$, and choose one element in each pair of complementary $l$-subsets. We then obtain a family $\left\{A_{1}, \ldots, A_{n}\right\}$ which satisfies (2). Now we set $\mathcal{F}=\left\{E_{1}, \ldots, E_{2 l}\right\}$, with $E_{i}=\left\{a_{j} ; i \in A_{j}\right\}$. The characterization (1) shows that $\mathcal{F}$ shatters every 2 -subset of $S$.

The proof of the following statement is straightforward.

Corollary 3 . If $\left(\begin{array}{c}2 l-1 \\ l-1\end{array}\right)<n \leq\left(\begin{array}{c}2 l+1 \\ l\end{array}\right)$, then $2 l \leq C(2, n) \leq 2 l+2$.

The upper bound can be slightly improved: One can prove that, if $\left(\begin{array}{c}2 l-1 \\ l-1\end{array}\right)<n \leq\left(\begin{array}{c}2 l \\ l-1\end{array}\right)$, then $2 l \leq C(2, n) \leq 2 l+1$.

Question 3. It seems that we have $C(2, n)=k$ if and only if $\left(\begin{array}{c}k-2 \\ \lfloor(k-1) / 2\rfloor-1\end{array}\right)<n \leq\left(\begin{array}{c}k-1 \\ \lfloor k / 2\rfloor-1\end{array}\right)$, where $\lfloor x\rfloor$ denotes the integer part of $x$. Is it true? Is it already known?

The first values are $C(2,2)=C(2,3)=4, C(2,4)=5, C(2,5)=\cdots=C(2,10)=6$. Computer seems to be useless, at least for a naive treatment. Already in order to obtain $C(2,11)=7$, we would have to verify that $C(2,11)>6$, i.e. to find, for each of the $\left(\begin{array}{c}2^{11} \\ 6\end{array}\right) \approx 10^{17}$ families $\mathcal{F}$ in $2^{S}$ some 2 -subset that is not shattered by the family. (Alternatively, in the dual statement, we have to check "only" $\left(\begin{array}{l}2^{6} \\ 11\end{array}\right) \approx 7.10^{11}$ families $\mathcal{S}$ in $2^{\mathcal{F}}$.)

The case $d \geq 3$. From now, we assume $n \geq 4$.

Proposition 4 . For all $3 \leq d<n$, we have $C(d, n) \leq \frac{2^{d}}{d !}(3 \log n)^{d}$.

The constant 3 can be improved. The proof below shows that, for all $a>1$ and all $n$ large enough, $C(d, n) \leq \frac{2^{d}}{d !}(a \log n)^{d}$.

Proof. Let $\mathcal{F}_{0} \subset 2^{S}$ be a minimal separating system of $S$, i.e. such that, for all $a, b \in S$ there exists $E_{a}^{b} \in \mathcal{F}_{0}$ which satisfies $b \notin E_{a}^{b} \ni a$. Since this amounts to choosing $\mathcal{F}_{0}$ minimal such that $\mathcal{S}=\varphi(S)$ is a Sperner family for $\mathcal{F}_{0}$, we know that $\left|\mathcal{F}_{0}\right|=N$ if and only if $\left(\begin{array}{c}N-1 \\ \lfloor(N-1) / 2\rfloor\end{array}\right)<$ $n \leq\left(\begin{array}{c}N \\ \lfloor N / 2\rfloor\end{array}\right)$, hence $N:=\left|\mathcal{F}_{0}\right| \leq 2+\log n+\frac{1}{2} \log \log n \leq 3 \log n$ since $n \geq 4$. We assume 
$N \geq 2$ in the sequel. Given two disjoint subsets $B$ and $C$ of $S$ such that $|B \cup C|=d$, the set $E_{B}^{C}=\bigcap_{c \in C}\left(\bigcup_{b \in B} E_{b}^{c}\right)$ contains $B$ and does not meet $C$. Let $\mathcal{F}$ be the collection of all such sets $E_{B}^{C}$; then $\mathcal{F}$ shatters all subsets of $S$ of cardinal at most $d$.

To estimate $|\mathcal{F}|$, we consider $\mathcal{F}_{k}$ the collection of all such sets $E_{B}^{C}$, with $|B|=k$ (and thus $|C|=d-k$ ). We have $\left|\mathcal{F}_{k}\right|=\left(\begin{array}{c}N \\ k\end{array}\right)\left(\begin{array}{c}N-k \\ d-k\end{array}\right)$ (with $\left.N=\left|\mathcal{F}_{0}\right|\right)$. Then we choose $\mathcal{F}=\bigcup_{k=0}^{d} \mathcal{F}_{k}$. We obtain $|\mathcal{F}| \leq \sum_{k=0}^{d}\left(\begin{array}{c}N \\ k\end{array}\right)\left(\begin{array}{c}N-k \\ d-k\end{array}\right)=\left(\begin{array}{c}N \\ d\end{array}\right) 2^{d} \leq \frac{2^{d}}{d !} N^{d} \leq \frac{2^{d}}{d !}(3 \log n)^{d}$.

Question 4 . Is $(\log n)^{\lfloor d / 2\rfloor\lfloor(d+1) / 2\rfloor}$ the right order of magnitude for $C(d, n)$ ?

By constructing auxiliary Sperner families from $\mathcal{S}$, it is possible to give a better lower bound for $C(d, n)$ than only $C(d, n) \geq C(2, n)$. For instance, in the case $d=3$, for all distinct $A, B, C \in \mathcal{S}$, we must have $A \cap B \nsubseteq \subset$. One can check that this implies that the family $\{A \cap B ; A, B \in \mathcal{S}\}$ is a Sperner family, therefore we obtain $\left(\begin{array}{c}n \\ 2\end{array}\right) \leq\left(\begin{array}{c}C(3, n) \\ {[C(3, n)\rfloor / 2}\end{array}\right)$. Unfortunately, this does not modify the order of magnitude. Already in this case $d=3$, we do not know whether $C(3, n)$ is of order $\log n,(\log n)^{2}$, or an intermediate order of magnitude. Another formulation is:

Question 5. Prove or disprove: There exists $C>0$ such that, for all $k \in \mathbb{N}$, if $\mathcal{F}$ is a finite set of cardinal $k$ and $\mathcal{S} \subseteq 2^{\mathcal{F}}$ satisfies $\forall A, B, C \in \mathcal{S}, A \cap B \nsubseteq C$, then $|\mathcal{S}| \leq C 2^{C \sqrt{k}}$.

\section{References}

[1] P. Borg, Intersecting families of sets and permutations: a survey. Int. J. Math. Game Theory Algebra 21 (2012) 543-559.

[2] G. Katona, On a conjecture of Erdös and a stronger form of Sperner's theorem. Studia Sci. Math. Hungar. 1 (1966) 59-63.

[3] D. Lubell, A short proof of Sperner's theorem, J. Combin Theory 1 (1966) 299.

[4] N. Sauer, On the density of families of sets, J. Combin. Theory 25 (1972) 80-83.

[5] S. Shelah, A combinatorial problem, stability and order for models and theories in infinite languages, Pacific J. Math. 41 (1972) 247-261.

[6] E. Sperner, Ein Satz über Untermenger einer endlichen Menge, Math. Zeitschrift 27 (1928) $544-548$.

[7] V. N. Vapnik and A. Y. Chervonenkis, On the uniform convergence of relative frequences of events to their probabilities Theory Probab. Appl. 16 (1971) 264-280. 